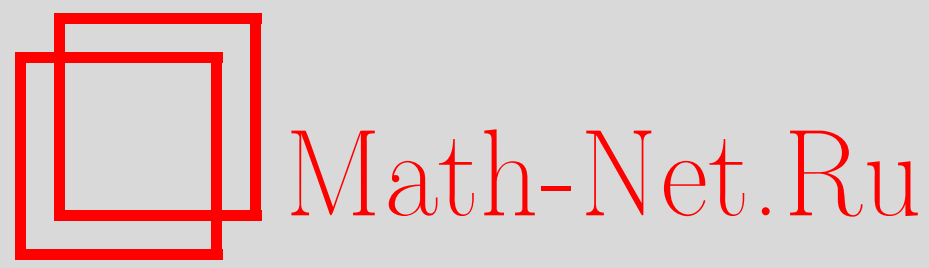

В. П. Маслов, О. Ю. Шведов, Приближенные несимметричные решения многочастичных уравнений и некоторые тождества для матрицы плотности, Матем. заметки, 1996, том 60, выпуск 3, 463-467

DOI: https://doi.org/10.4213/mzm1852

Использование Общероссийского математического портала MathNet.Ru подразумевает, что вы прочитали и согласны с пользовательским соглашением

http://www . mathnet.ru/rus/agreement

Параметры загрузки:

IP : 34.227 .88 .159

26 апреля 2023 г., 17:06:05

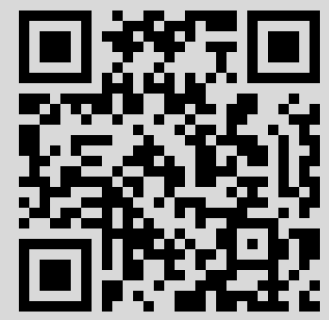




\section{ПРИБЛИЖЕННЫЕ НЕСИММЕТРИЧНЫЕ РЕШЕНИЯ МНОГОЧАСТИЧНЫХ УРАВНЕНИЙ И НЕКОТОРЫЕ ТОЖДЕСТВА ДЛЯ МАТРИЦЫ ПЛОТНОСТИ}

\section{В.П. Маслов, О.Ю. Шведов}

В работах [1]-[4] рассмотрено построение аппроксимаций при $N \rightarrow \infty$ для симметричных функций $N$ аргументов по норме пространства $L^{2}\left(X^{N}\right)$, где $X-$ пространство с мерой. В частности, в работе [1] построены приближенные спектральные серии

$$
E^{(\nu)}=N \mathscr{E}_{0}+\sum_{k=1}^{\infty} \beta_{k} \nu_{k}+O\left(\frac{1}{N}\right), \quad \nu=\left(\nu_{1}, \nu_{2}, \ldots\right), \quad \nu_{k} \in\{0,1,2, \ldots\},
$$

$N$-частичного оператора Шрёдингера $H_{N}$, действующего в $L^{2}\left(X^{N}\right)$.

Аналогичным образом можно построить [3] приближенные стационарные решения многочастичного уравнения Вигнера

$$
\left[H_{N}, \rho\right]=\lambda \rho,
$$

где $\rho$ - оператор в $L^{2}\left(X^{N}\right)$. Уравнение (2) имеет приближенные решения $\rho^{(\mu \nu)}$ вида

$$
\rho^{(\mu \nu)} f=\psi^{(\mu)}\left(\psi^{(\nu)}, f\right), \quad f \in L^{2}(X),
$$

где $\psi^{(\mu)}, \psi^{(\nu)}$ - асимптотические собственные функции оператора $H_{N}$, отвечающие собственным значениям $E^{(\mu)}, E^{(\nu)}$. Величина $\lambda$ имеет вид

$$
\lambda^{(\mu \nu)}=E^{(\mu)}-E^{(\nu)}+O\left(\frac{1}{N}\right) .
$$

Оказывается, что метод работы [3] дает, помимо спектра (3), другие асимптотические собственные значения задачи (2). В этой работе мы покажем, что они выражаются через приближенные собственные значения оператора $H_{N}$, которым отвечают собственные функции, не являющиеся симметричными по всем $N$ аргументам. Мы найдем также связь собственных векторов задачи (2) и оператора $H_{N}$.

Не ограничивая общности, будем считать $X=\mathbb{N}$. Напомним результат [1]. Рассмотрим оператор $H_{N}$ вида

$$
\begin{gathered}
\left(H_{N} \psi\right)_{I_{1} \ldots I_{N}}=\sum_{k=0}^{L} N^{1-k} \sum_{1 \leqslant i_{1}<\cdots<i_{k} \leqslant N} \sum_{J_{i_{1}}, \ldots, J_{i_{k}}=1}^{\infty} \\
H_{I_{i_{1}} \ldots I_{i_{k}} ; J_{i_{1}} \ldots J_{i_{k}}}^{(k)} \psi_{I_{1} \ldots I_{i_{1}-1} J_{i_{1}} I_{i_{1}+1} \ldots I_{i_{k}-1} J_{i_{k}} I_{i_{k}+1} \ldots I_{N}},
\end{gathered}
$$

где $I_{1}, \ldots, I_{N}=\overline{1, \infty}, \psi: \mathbb{N} \times \cdots \times \mathbb{N} \rightarrow \mathbb{C}, L \in \mathbb{N}, \sum_{I_{1}, \ldots, I_{N} \in \mathbb{N}}\left|\psi_{I_{1} \ldots I_{N}}\right|^{2}<\infty$. Обозначим

$$
H\left(\varphi^{*}, \varphi\right)=\sum_{k=0}^{L} \frac{1}{k !} \sum_{I_{1}, \ldots, I_{k}, J_{1}, \ldots, J_{k} \in \mathbb{N}} H_{I_{1} \ldots I_{k} ; J_{1} \ldots J_{k}}^{(k)} \varphi_{I_{1}}^{*} \cdots \varphi_{I_{k}}^{*} \varphi_{J_{1}} \cdots \varphi_{J_{k}},
$$


$\varphi: \mathbb{N} \rightarrow \mathbb{C}$. Спектральные серии оператора $H_{N}$ отвечают решениям уравнения типа Хартри

$$
\Omega \varphi_{I}=\frac{\partial H}{\partial \varphi_{I}^{*}}
$$

Асимптотические собственные значения $H_{N}$ выражаются через спектр системы в вариациях

$$
\begin{aligned}
\left(\beta_{l}+\Omega\right) G_{M l} & =\sum_{J=1}^{\infty}\left(\frac{\partial^{2} H}{\partial \varphi_{M} \partial \varphi_{J}^{*}} G_{J l}+\frac{\partial^{2} H}{\partial \varphi_{M} \partial \varphi_{J}} F_{J l}\right) \\
-\left(\beta_{l}-\Omega\right) F_{M l} & =\sum_{J=1}^{\infty}\left(\frac{\partial^{2} H}{\partial \varphi_{M}^{*} \partial \varphi_{J}^{*}} G_{J l}+\frac{\partial^{2} H}{\partial \varphi_{M}^{*} \partial \varphi_{J}} F_{J l}\right) .
\end{aligned}
$$

Пусть $\beta_{l}, F_{M l}, G_{M l}, l=\overline{0, \infty},-$ решение системы (6), удовлетворяющее условиям

$$
\begin{gathered}
F^{T} G=G^{T} F, \quad F_{M 0}=-\varphi_{M}, \quad G_{M 0}=\varphi_{M}^{*}, \\
\sum_{M=1}^{\infty}\left(G_{M \alpha}^{*} G_{M \beta}-F_{M \alpha}^{*} F_{M \beta}\right)=\delta_{\alpha \beta}, \quad \alpha, \beta=\overline{1, \infty}
\end{gathered}
$$

матрица $G$ обратима, ряд $\sum_{M \in \mathbb{N}} \sum_{l=0}^{\infty}\left|F_{M l}\right|^{2}$ сходится. Обозначим

$$
\begin{aligned}
& R_{I J}=\left(F G^{-1}\right)_{I J}, \quad M_{I J}=R_{I J}+\varphi_{I} \varphi_{J}, \\
& \mathscr{E}_{0}=H\left(\varphi^{*}, \varphi\right)+\frac{1}{2 N} \sum_{I, J=1}^{\infty} \frac{\partial^{2} H}{\partial \varphi_{I} \partial \varphi_{J}} R_{I J}, \\
& \psi_{I_{1} \ldots I_{N}}^{(0) N}=\sum_{l=0}^{[N / 2]} \frac{1}{(2 N)^{l} l !} \sum_{1 \leqslant i_{1} \neq \cdots \neq i_{2 l} \leqslant N} M_{I_{i_{1}} I_{i_{2}}} \cdots M_{I_{i_{2 l-1}} I_{i_{2 l}}} \prod_{\substack{i \neq i_{s} \\
s=1,2 l}} \varphi_{I_{i}} .
\end{aligned}
$$

Рассмотрим операторы $A_{\alpha}^{+}, \alpha=\overline{1, \infty}$, вида

$$
\left(A_{\alpha}^{+} \psi\right)_{I_{1} \ldots I_{N}}=\frac{1}{\sqrt{N}} \sum_{i=1}^{N} T_{I_{i} J_{i}}^{\alpha} \psi_{I_{1} \ldots I_{i-1} J_{i} I_{i+1} \ldots I_{N}}
$$

где

$$
T_{I J}^{\alpha}=\left(G_{I \alpha}^{*}-\varphi_{I} \sum_{P=1}^{\infty} G_{P \alpha}^{*} \varphi_{P}^{*}\right) \varphi_{J}^{*}-\varphi_{I}\left(F_{J \alpha}^{*}-\varphi_{J}^{*} \sum_{P=1}^{\infty} F_{P \alpha}^{*} \varphi_{P}\right)
$$

Обозначим

$$
\psi^{(\nu) N}=\left(A_{1}^{+}\right)^{\nu_{1}}\left(A_{2}^{+}\right)^{\nu_{2}} \cdots \psi^{(0) N} .
$$

Теорема 1. Bиполнено соотношение

$$
\left\|\psi^{(\nu) N}\right\|_{L^{2}}=O(1), \quad\left\|\left(H_{N}-E^{(\nu)}\right) \psi^{(\nu) N}\right\|_{L^{2}}=O\left(\frac{1}{\sqrt{N}}\right) .
$$

Сопоставим оператору $\rho$ его ядро $\rho_{I_{1} \ldots I_{N} ; J_{1} \ldots J_{N}}$, будем обозначать

$$
\|\rho\|_{L^{2}}=\sum_{I_{1}, \ldots, I_{N}, J_{1}, \ldots, J_{N} \in \mathbb{N}}\left|\rho_{I_{1} \ldots I_{N} ; J_{1} \ldots J_{N}}\right|^{2} .
$$


Оператор в левой части уравнения (2), действующий на $\rho$, относится к типу (4). Поэтому спектральные серии этого оператора могут быть найдены с помощью теоремы 1 .

Обозначим

$$
\begin{aligned}
& \mathscr{H}\left(\Phi^{*}, \Phi\right)=\sum_{k=0}^{L} \frac{1}{k !} \sum_{I_{1}, \ldots, I_{k}, J_{1}, \ldots, J_{k}, L_{1}, \ldots, L_{k}, M_{1}, \ldots, M_{k}=1}^{\infty} \\
& \quad\left[H_{I_{1} \ldots I_{k} ; J_{1} \ldots J_{k}}^{(k)} \delta_{L_{1} M_{1}} \cdots \delta_{L_{k} M_{k}}-H_{M_{1} \ldots M_{k} ; L_{1} \ldots L_{k}}^{(k)} \delta_{I_{1} J_{1}} \cdots \delta_{I_{k} J_{k}}\right] \\
& \quad \times \Phi_{I_{1} L_{1}}^{*} \cdots \Phi_{I_{k} L_{k}}^{*} \Phi_{J_{1} M_{1}} \cdots \Phi_{J_{k} M_{k}}, \quad \Phi: \mathbb{N} \times \mathbb{N} \rightarrow \mathbb{C} .
\end{aligned}
$$

ЛЕмма 1. Функиия $\Phi_{I J}=\varphi_{I} \varphi_{J}^{*}$ удовлетворяет уравнению

$$
\frac{\partial \mathscr{H}}{\partial \Phi_{I J}^{*}}=0 .
$$

Отметим, что уравнение (9) является аналогом уравнения (5), а аналогом системы (6) является система

$$
\begin{aligned}
\gamma_{l m} \mathscr{Y}_{M K l m} & =\sum_{I, J=1}^{\infty}\left(\frac{\partial^{2} \mathscr{H}}{\partial \Phi_{M K} \partial \Phi_{I J}^{*}} \mathscr{Y}_{I J l m}+\frac{\partial^{2} \mathscr{H}}{\partial \Phi_{M K} \partial \Phi_{I J}} \mathscr{F}_{I J l m}\right) \\
-\gamma_{l m} \mathscr{F}_{M K l m} & =\sum_{I, J=1}^{\infty}\left(\frac{\partial^{2} \mathscr{H}}{\partial \Phi_{M K}^{*} \partial \Phi_{I J}^{*}} \mathscr{Y}_{I J l m}+\frac{\partial^{2} \mathscr{H}}{\partial \Phi_{M K}^{*} \partial \Phi_{I J}} \mathscr{F}_{I J l m}\right) .
\end{aligned}
$$

Обозначим через $W_{I J}$ матрицу вида

$$
\begin{aligned}
W_{I J}= & \sum_{k=1}^{L} \frac{1}{(k-1) !} \sum_{I_{1}, \ldots, I_{k-1}, J_{1}, \ldots, J_{k-1} \in \mathbb{N}} \varphi_{I_{1}}^{*} \cdots \varphi_{I_{k-1}}^{*} \\
& \times H_{I I_{1} \ldots I_{k-1} ; J J_{1} \ldots J_{k-1}}^{(k)} \varphi_{J_{1}} \cdots \varphi_{J_{k-1}}
\end{aligned}
$$

а через $\varepsilon^{(m)}$ и $\chi_{J}^{(m)}$ - ее собственные значения и собственные функции

$$
\sum_{J=1}^{\infty} W_{I J} \chi_{J}^{(m)}=\varepsilon^{(m)} \chi_{I}^{(m)}, \quad m=\overline{0, \infty}
$$

причем

$$
\chi_{J}^{(0)}=\varphi_{J}, \quad \varepsilon^{(0)}=\Omega .
$$

Лемма 2. Система (10) имеет решения вида

$$
\begin{array}{ccc}
\mathscr{F}_{M K \alpha \rho}=0, \quad \mathscr{Y}_{M K \alpha \rho}=\chi_{M}^{(\alpha) *} \chi_{K}^{(\rho)}, \quad \alpha, \rho=\overline{1, \infty}, & \gamma_{\alpha \rho}=\varepsilon^{(\alpha)}-\varepsilon^{(\rho)}, \\
\mathscr{F}_{M K 0 \rho}=\varphi_{M} F_{K \rho}^{*}, \quad \mathscr{Y}_{M K 0 \rho}=\varphi_{M}^{*} G_{K \rho}^{*}, & \gamma_{0 \rho}=-\beta_{\rho}, \\
\mathscr{F}_{M K \alpha 0}=F_{M \alpha} \varphi_{K}^{*}, & \mathscr{Y}_{M K \alpha 0}=G_{M \alpha} \varphi_{K}, & \gamma_{\alpha 0}=\beta_{\alpha}, \\
\mathscr{F}_{M K 00}=-\varphi_{M} \varphi_{K}^{*}, & \mathscr{Y}_{M K 00}=\varphi_{M}^{*} \varphi_{K}, & \gamma_{00}=0 .
\end{array}
$$


Обозначим

$$
\begin{aligned}
& \mathscr{M}_{I_{1} J_{1} ; I_{2} J_{2}}=M_{I_{1} I_{2}} \varphi_{J_{1}}^{*} \varphi_{J_{2}}^{*}+\varphi_{I_{1}} \varphi_{I_{2}} M_{I_{1} I_{2}}^{*} \\
& \left(\mathscr{A}_{m n}^{+} \rho\right)_{I_{1} \ldots I_{N}, J_{1} \ldots J_{N}}=\frac{1}{\sqrt{N}} \sum_{i=1}^{N} L_{I_{i} J_{i} ; M_{i} K_{i}}^{(m n)} \rho_{I_{1} \ldots M_{i} \ldots I_{N} ; J_{1} \ldots K_{i} \ldots J_{N}} \text {, } \\
& L_{I J ; M K}^{(m n)}=\left(\mathscr{Y}_{I J m n}^{*}-\sum_{P, Q=1}^{\infty} \Phi_{I J} \Phi_{P Q}^{*} \mathscr{Y}_{P Q m n}^{*}\right) \Phi_{M K}^{*} \\
& -\Phi_{I J}\left(\mathscr{F}_{M K m n}^{*}-\sum_{P, Q=1}^{\infty} \Phi_{M K}^{*} \Phi_{P Q} \mathscr{F}_{P Q m n}^{*}\right), \\
& \nu=\left(\nu_{1}, \nu_{2}, \ldots\right), \quad \mu=\left(\mu_{1}, \mu_{2}, \ldots\right), \quad \varkappa=\left(\varkappa_{11}, \varkappa_{12}, \ldots, \varkappa_{21}, \varkappa_{22}, \ldots\right) \text {, } \\
& \rho^{(\mu \nu \varkappa)}=\prod_{\alpha=1}^{\infty}\left(\mathscr{A}_{\alpha 0}^{+}\right)^{\mu_{\alpha}} \prod_{\beta=1}^{\infty}\left(\mathscr{A}_{0 \beta}^{+}\right)^{\nu_{\beta}} \prod_{\rho, \sigma=1}^{\infty}\left(\mathscr{A}_{\rho \sigma}^{+}\right)^{\varkappa \rho \sigma} \rho^{(0)}, \\
& \rho_{I_{1} \ldots I_{N} ; J_{1} \ldots J_{N}}^{(0)}=\sum_{l=0}^{[N / 2]} \frac{1}{(2 N)^{l} l !} \sum_{1 \leqslant i_{1} \neq \cdots \neq i_{2 l} \leqslant N} \mathscr{M}_{I_{i_{1}} J_{i_{1}} ; I_{i_{2}} J_{i_{2}} \ldots} \\
& \times \mathscr{M}_{I_{i_{2 l-1}} J_{i_{2 l-1}} ; I_{2 l}} J_{i_{2 l}} \prod_{i \neq i_{s}} \Phi_{I_{i} J_{i}} \\
& \lambda^{(\mu \nu \varkappa)}=\sum_{\alpha=1}^{\infty} \beta_{\alpha}\left(\mu_{\alpha}-\nu_{\alpha}\right)+\sum_{\rho, \sigma=1}^{\infty} \varkappa_{\rho \sigma}\left(\varepsilon^{(\rho)}-\varepsilon^{(\sigma)}\right) .
\end{aligned}
$$

Из теоремы 1 вытекает

Теорема 2. Bыполнено соотношение

$$
\left\|\rho^{(\mu \nu \varkappa)}\right\|_{L^{2}}=O(1), \quad\left\|\left[H_{N}, \rho^{(\mu \nu \varkappa)}\right]-\lambda^{(\mu \nu \varkappa)} \rho^{(\mu \nu \varkappa)}\right\|_{L^{2}}=O\left(\frac{1}{\sqrt{N}}\right) .
$$

Рассмотрим связь функций (7) и матриц плотности (11).

Теорема 3. Пусть $\varkappa_{\rho \sigma}=0, \rho, \sigma=\overline{1, \infty}$. Тољда

$$
\left\|\rho^{(\mu \nu 0)}-\rho^{(\mu \nu)}\right\|_{L^{2}} \underset{\rightarrow \rightarrow \infty}{ } 0 .
$$

Отметим, что функции $\rho_{I_{1} \ldots I_{N} ; J_{1} \ldots J_{N}}^{(\mu \nu)}$ обладают свойством симметрии отдельно по $I_{1}, \ldots, I_{N}$ и отдельно по $J_{1}, \ldots, J_{N}$. Оказывается, что при $\varkappa \neq 0$ построенные асимптотики таким свойством не обладают.

ЛЕмма 3. Пусть функиия $\rho_{I_{1} \ldots I_{N} ; J_{1} \ldots J_{N}}^{(\mu \nu \varkappa м м е т р и ч н а ~ о т д е л ь н о ~ п о ~} I_{1}$, $\ldots, I_{N}$ и отдельно по $J_{1}, \ldots, J_{N}$. Тогда $\varkappa=0$.

Таким образом, для построения матрицы плотности $\rho$ при $\varkappa \neq 0$ необходимо использовать несимметричные собственные функции гамильтониана $H_{N}$.

Рассмотрим функции вида

$$
\psi_{I_{1} \ldots I_{N}}^{\left\{\alpha_{1} \ldots \alpha_{p}\right\}(\mu)}=\chi_{I_{1}}^{\left(\alpha_{1}\right)} \cdots \chi_{I_{p}}^{\left(\alpha_{p}\right)} \psi_{I_{p+1} \ldots I_{N}}^{(\mu) N-p}, \quad \alpha_{j}=\overline{1, \infty}
$$


Теорема 4. Виполнено соотношение

$$
\begin{gathered}
\left\|\psi^{\left\{\alpha_{1} \ldots \alpha_{p}\right\}(\mu)}\right\|_{L^{2}}=O(1) \\
\left\|\left(H_{N}-E^{(\mu)}-\sum_{j=1}^{p}\left(\varepsilon^{\left(\alpha_{j}\right)}-\Omega\right)\right) \psi^{\left\{\alpha_{1} \ldots \alpha_{p}\right\}(\mu)}\right\|_{L^{2}}=O\left(\frac{1}{\sqrt{N}}\right) .
\end{gathered}
$$

Обозначим через $P$ оператор симметризации вида

$$
(P \rho)_{I_{1} \ldots I_{N} ; J_{1} \ldots J_{N}}=\frac{1}{N !} \sum_{1 \leqslant \sigma_{1} \neq \cdots \neq \sigma_{N} \leqslant N} \rho_{I_{\sigma_{1}} \ldots I_{\sigma_{N}} ; J_{\sigma_{1}} \ldots J_{\sigma_{N}}} .
$$

Рассмотрим матрицу плотности

$$
\widetilde{\rho}_{I_{1} \ldots I_{N} ; J_{1} \ldots J_{N}}^{(\mu \nu \varkappa)}=P \psi_{I_{1} \ldots I_{N}}^{\left\{\alpha_{1} \ldots \alpha_{p}\right\}(\mu)} \psi_{J_{1} \ldots J_{N}}^{*\left\{\beta_{1} \ldots \beta_{p}\right\}(\nu)}
$$

где $\varkappa_{i j}$ - количество пар $(i, j)$ в наборе из $p$ пар $\left(\alpha_{1}, \beta_{1}\right), \ldots,\left(\alpha_{p}, \beta_{p}\right)$.

Лемма 4. Справедливы свойства

$$
\begin{gathered}
\left\|\widetilde{\rho}^{(\mu \nu \varkappa)}\right\|_{L^{2}}=O\left(\frac{1}{\sqrt{N^{p}}}\right) \\
\left\|\left[H_{N}, \widetilde{\rho}^{(\mu \nu \varkappa)}\right]-\lambda^{(\mu \nu \varkappa)} \widetilde{\rho}^{(\mu \nu \varkappa)}\right\|_{L^{2}}=O\left(\frac{1}{\sqrt{N^{p+1}}}\right) .
\end{gathered}
$$

Оказьвается, что матрица плотности (16) после умножения на нормировочньй фактор $\sqrt{N^{p}} / p$ ! приближенно совпадает с (11).

Теорема 5. Выполняется соотношение

$$
\left\|\rho^{(\mu \nu \varkappa)}-\frac{\sqrt{N^{p}}}{p !} \widetilde{\rho}^{(\mu \nu \varkappa)}\right\|_{L^{2} N \rightarrow \infty} 0 .
$$

Таким образом, мы выразили приближенные собственные функции задачи (2) через, вообще говоря, несимметричные асимптотические собственные функции опеpaтора $H_{N}$.

Московский государственный университет

Поступило

им. М.В. Ломоносова

30.04 .96

\section{СПИСОК ЦИТИРОВАННОЙ ЛИТЕРАТУРЫ}

1. Маслов В.П., Шведов О. Ю. // ТМФ. 1994. Т. 98. № 2. С. 266-288. 2. Maslov V.P., Shvedov O. Yu. // Russian J. Math. Phys. 1994. V. 2. № 2. P. 217-234. 3. Maslov V. P., Shvedov O. Yu. // Russian J. Math. Phys. 1995. V. 3. № 1. P. 65-80. 4. Maslov V. P., Shvedov O. Yu. Geometric quantization in Fock space // Advances in Soviet Mathematics. Berezin memorial volume. Providence: AMS, 1996. 